\title{
Assisted state discrimination without entanglement
}

\author{
Bo Li, ${ }^{1,2, \text {, }}$ Shao-Ming Fei, ${ }^{3,4}$ Zhi-Xi Wang, ${ }^{3}$ and Heng Fan ${ }^{1, \text {, }}$ \\ ${ }^{1}$ Institute of Physics, Chinese Academy of Sciences, Beijing 100190, China \\ ${ }^{2}$ Department of Mathematics and Computer, Shangrao Normal University, Shangrao 334001, China \\ ${ }^{3}$ School of Mathematical Sciences, Capital Normal University, Beijing 100048, China \\ ${ }^{4}$ Max-Planck-Institute for Mathematics in the Sciences, 04103 Leipzig, Germany
}

\begin{abstract}
It is shown that the dissonance, a quantum correlation which is equal to quantum discord for separable state, is required for assisted optimal state discrimination. We find that only one side discord is required in the optimal process of assisted state discrimination, while another side discord and entanglement is not necessary. We confirm that the quantum discord, which is asymmetric depending on local measurements, is a resource for assisted state discrimination. With the absence of entanglement, we give the necessary and sufficient condition for vanishing one side discord in assisted state discrimination for a class of $d$ nonorthogonal states. As a byproduct, we find that the positive-partial-transposition (PPT) condition is the necessary and sufficient condition for the separability of a class of $2 \times d$ states.
\end{abstract}

PACS numbers: 03.67.-a, 03.65.Ud, 03.65.Yz

\section{INTRODUCTION}

Entanglement is regarded as a key resource in quantum information processing such as teleportation and superdense coding, etc.[1]. On the other hand, it is shown that a deterministic quantum computation with one qubit(DQC1) [3] can be carried out without entanglement. While the quantum discord [4 [8], another type of quantum correlation, might be the reason for the advantage of this algorithm that surpasses the corresponding classical algorithms. Besides quantum entanglement, there are also many quantum nonlocal properties which can be manifested by separable states or separable operations [2]. It is thus reasonable to assume that the quantumness correlation can be viewed from different aspects. Recently much efforts have been devoting to studying various measures of nonclassical correlation, see for example Refs. 27 35]. Quantum discord, which is our main concern in this paper, is recently pointed out to have operational interpretations, for example in terms of the quantum state merging protocol 21] and as entanglement by an activation protocol or by measurement [27, 28].

Quantum discord [4, 5] is measured by the difference between the mutual information and the maximal conditional mutual information obtained by local measurement. Explicitly, let us consider a bipartite quantum state $\rho_{A B}$, the "right" quantum discord is given by [4, 5],

$D_{B}\left(\rho_{A B}\right)=I\left(\rho_{A B}\right)-\sup _{E_{k}}\left\{S\left(\rho_{A}\right)-\sum_{k} p_{A \mid k} S\left(\rho_{A \mid k}\right)\right\},(1)$

where $S(\rho)$ is the von Neumann entropy and $I\left(\rho_{A B}\right)=$ $S\left(\rho_{A}\right)+S\left(\rho_{B}\right)-S\left(\rho_{A B}\right)$ is the quantum mutual information, and $p_{A \mid k}=\operatorname{Tr}\left(\mathbb{1}_{A} \otimes E_{k} \rho\right), \rho_{A \mid k}=\operatorname{Tr}_{B}\left(\mathbb{1}_{A} \otimes\right.$

*Electronic address: libo@iphy.ac.cn

†Electronic address: hfan@iphy.ac.cn
$\left.E_{k} \rho\right) / p_{A \mid k}$, the supreme is taken over all the von Neumann measurement sets $\left\{E_{k}\right\}$ on system $B$. Similarly, $D_{A}\left(\rho_{A B}\right)$ refers to the "left" discord and is given by

$$
D_{A}\left(\rho_{A B}\right)=I\left(\rho_{A B}\right)-\sup _{E_{k}}\left\{S\left(\rho_{B}\right)-\sum_{k} p_{B \mid k} S\left(\rho_{B \mid k}\right)\right\},(2)
$$

with $p_{B \mid k}=\operatorname{Tr}\left(E_{k} \otimes \mathbb{1}_{B} \rho\right), \rho_{B \mid k}=\operatorname{Tr}_{A}\left(\left(E_{k} \otimes \mathbb{1}_{B} \rho\right) / p_{B \mid k}\right.$. Note that the difference between those two discords is that the measurement is performed on party $A$ or on party $B$, respectively. It is thus expected that this definition of quantum discord is not symmetric with respect to $A$ and $B$.

Due to the supreme in the definition (11), quantum discord does not have analytic or operational expression in general. However, explicit expression of discord has been found for cases like Bell-diagonal state 13, 14] and subclass of so-called $X$ states 15 17]. Numerical results for general two-qubit states are also presented in [22]. Moreover, the quantum discord can be related to the entanglement of formation (EOF) [23]. This relation can be used to calculate the quantum discord for the ranktwo states 26]. Nevertheless, zero discord can be easily found by a necessary and sufficient condition pointed out in Ref.[20]. If $\rho_{A B}$ is a quantum state of $d_{A} \times d_{B}$ dimensional bipartite quantum systems, from [20] $\rho_{A B}$ can be written in diagonal form $\rho_{A B}=\sum_{n=1}^{L} c_{n} S_{n} \otimes F_{n}$, where $\left\{S_{n}\right\},\left\{F_{m}\right\},\left(n=1, \cdots, d_{A}^{2}, m=1, \cdots, d_{B}^{2}\right)$ are the bases of the respective local spaces, $L$ is the rank of $\rho[20]$. Then the necessary and sufficient condition of zero "left" discord is

$$
\left[S_{i}, S_{j}\right]=0, i, j=1, \cdots, L .
$$

Similarly, the necessary and sufficient condition of zero "right" discord is

$$
\left[F_{i}, F_{j}\right]=0, i, j=1, \cdots, L .
$$

It is shown that almost all quantum states have nonzero discord 24, 25]. 
A unified view of quantum correlation based on relative entropy was introduced in 36]. Quantum dissonance is a kind of quantum correlation of separable states. As the closest state to a separable state $\rho$ as measured by relative entropy is the state $\rho$ itself, the dissonance is just the quantum discord in this case.

It is remarkable that dissonance is found to be useful in assisted state discrimination recently [1]. Note that dissonance is the quantum discord in the optimal case. In this paper, we further find that actually only one side quantum discord appears in the optimal process, while discord of another side can be zero. Hence the role of quantum discord played in assisted state discrimination might be quite different for different parties. We show clearly that the quantum discord is really a useful resource which can be used in assisted state discrimination. We also extend the study of discord for assisted state discrimination to more general cases.

The paper is organized as follows. In Sec. III we first give a brief review of the model in assisted state discrimination. We then show that only one side discord is necessary in the optimal case. In Sec. [II and Sec. [IV we generalize the model to discriminate a class of $d$ nonorthogonal states, in which we still find that one-side discord is required in the process. Sec. $\nabla$ is the summary.

\section{UNAMBIGUOUS DISCRIMINATION OF TWO NONORTHOGONAL STATES}

Following the model of assisted state discrimination in Ref. 9 11], consider that a qubit is randomly prepared in one of the two nonorthogonal states $\left|\psi_{+}\right\rangle$or $\left|\psi_{-}\right\rangle$with a priori probabilities $p_{+}$and $p_{-}$with $p_{+}+p_{-}=1$. Our aim is to discriminate the two states $\left|\psi_{+}\right\rangle$or $\left|\psi_{-}\right\rangle$. The system is coupled to an auxiliary qubit $A$ by a joint unitary transformation $U$ such that

$$
\begin{aligned}
U\left|\psi_{+}\right\rangle|k\rangle_{a} & =\sqrt{1-\left|\alpha_{+}\right|^{2}}|+\rangle|0\rangle_{a}+\alpha_{+}|0\rangle|1\rangle_{a}, \\
U\left|\psi_{-}\right\rangle|k\rangle_{a} & =\sqrt{1-\left|\alpha_{-}\right|^{2}}|-\rangle|0\rangle_{a}+\alpha_{-}|0\rangle|1\rangle_{a},
\end{aligned}
$$

where $|k\rangle_{a}$ is an auxiliary state with orthonormal basis $\left\{|0\rangle_{a},|1\rangle_{a}\right\},| \pm\rangle \equiv(|0\rangle \pm|1\rangle) / \sqrt{2}$ are the orthonormal states of the system that can be discriminated. For convenience, we adopt the symbols used in Ref. [11]. A priori fixed overlap is, $\left\langle\psi_{+} \mid \psi_{-}\right\rangle=\alpha=|\alpha| e^{i \theta}=\alpha_{+}^{*} \alpha_{-}$, where $\alpha_{+}^{*}$ is the complex conjugate of $\alpha_{+}, \theta$ is the phases of $\alpha$. The mixed state we consider in discrimination is given by

$$
\begin{aligned}
\rho_{\left|\alpha_{+}\right|}= & p_{+} U\left(\left|\psi_{+}\right\rangle\left\langle\psi_{+}|\otimes| k\right\rangle_{a}\langle k|\right) U^{\dagger} \\
& +p_{-} U\left(\left|\psi_{-}\right\rangle\left\langle\psi_{-}|\otimes| k\right\rangle_{a}\langle k|\right) U^{\dagger} .
\end{aligned}
$$

By performing a von Neumann measurement on the auxiliary system by basis, $\left\{|0\rangle_{a}\langle 0|,| 1\rangle_{a}\langle 1|\right\}$, the auxiliary state in (6) will collapse to either $\left\{|0\rangle_{a}\langle 0|\right\}$ or $\left\{|1\rangle_{a}\langle 1|\right\}$. In case the system collapses to $\left\{|0\rangle_{a}\langle 0|\right\}$, we will discriminate successfully the original state since we can distinguish deterministically the states $| \pm\rangle$ as in (5). The success probability is given by

$$
P\left(\left|\alpha_{+}\right|\right)=1-p_{-} \frac{|\alpha|^{2}}{\left|\alpha_{+}\right|^{2}}-p_{+}\left|\alpha_{+}\right|^{2} .
$$

For general $\left|\alpha_{+}\right|$, the probability is neither 0 nor 1 .

We shall use the PPT criterion [18] to characterize the separability of $\rho_{\left|\alpha_{+}\right|}$. For $2 \times 2$ and $2 \times 3$ systems, the PPT condition is both necessary and sufficient for the separability of quantum states [19]. From the PPT criterion, $\rho_{\left|\alpha_{+}\right|}$is separable if and only if its partial transposed matrix $\rho_{\left|\alpha_{+}\right|}^{T_{A}}$ has a non-negative spectrum. Hence the determinant $D$ of $\rho_{\left|\alpha_{+}\right|}^{T_{A}}$ should be non-negative either. By direct calculation we have

$$
\begin{aligned}
D & =-\left(p_{+} \frac{1-\left|\alpha_{+}\right|^{2}}{2}+p_{-} \frac{1-\left|\alpha_{-}\right|^{2}}{2}\right) \times\left(p_{+}\left|\alpha_{+}\right|^{2}+\right. \\
& \left.p_{-}\left|\alpha_{-}\right|^{2}\right) \times\left|p_{+} \sqrt{\frac{1-\left|\alpha_{+}\right|^{2}}{2}} \alpha_{+}-p_{-} \sqrt{\frac{1-\left|\alpha_{-}\right|^{2}}{2}} \alpha_{-}\right|^{2} .
\end{aligned}
$$

$D \geq 0$ implies that

$$
p_{+} \sqrt{\frac{1-\left|\alpha_{+}\right|^{2}}{2}} \alpha_{+}=p_{-} \sqrt{\frac{1-\left|\alpha_{-}\right|^{2}}{2}} \alpha_{-},
$$

that is,

$$
p_{+} \sqrt{\frac{1-\left|\alpha_{+}\right|^{2}}{2}}\left|\alpha_{+}\right|^{2}=p_{-} \sqrt{\frac{1-\left|\alpha_{-}\right|^{2}}{2}} \alpha,
$$

where $\alpha=\alpha_{+}^{*} \alpha_{-}$. From (9) we know that $\alpha$ must be a real number. Eq. (9) is a necessary condition for the separability of $\rho_{\left|\alpha_{+}\right|}$and is actually also a sufficient condition. It is in fact the same as the Eq.(7) in [11].

That the condition (8) is also a sufficient condition for separability can be seen from the following separable form of $\rho_{\left|\alpha_{+}\right|}$,

$$
\begin{aligned}
\rho_{\left|\alpha_{+}\right|}= & \left(1-p_{+}\left|\alpha_{+}\right|^{2}-p_{-}\left|\alpha_{-}\right|^{2}\right) \rho_{1}^{S} \otimes|0\rangle_{a}\langle 0|+ \\
& \left(p_{+}\left|\alpha_{+}\right|^{2}+p_{-}\left|\alpha_{-}\right|^{2}\right)|0\rangle\langle 0| \otimes \rho_{2}^{A},
\end{aligned}
$$

where $\rho_{1}^{S}$ and $\rho_{2}^{A}$ are the density matrices of the principal system and the auxiliary system respectively,

$$
\begin{aligned}
\rho_{1}^{S}= & \frac{1}{1-p_{+}\left|\alpha_{+}\right|^{2}-p_{-}\left|\alpha_{-}\right|^{2}}\left(p_{+}\left(1-\left|\alpha_{+}\right|^{2}\right)|+\rangle\langle+|\right. \\
& \left.+p_{-}\left(1-\left|\alpha_{-}\right|^{2}\right)|-\rangle\langle-|\right), \\
\rho_{2}^{A}= & \frac{1}{p_{+}\left|\alpha_{+}\right|^{2}+p_{-}\left|\alpha_{-}\right|^{2}}\left(\left(p_{+}\left|\alpha_{+}\right|^{2}+p_{-}\left|\alpha_{-}\right|^{2}\right)|1\rangle_{a}\langle 1|\right. \\
& +\sqrt{2} p_{+} \alpha_{+} \sqrt{1-\left|\alpha_{+}\right|^{2}}|1\rangle_{a}\langle 0|+ \\
& \left.\sqrt{2} p_{+} \alpha_{+}^{*} \sqrt{1-\left|\alpha_{+}\right|^{2}}|0\rangle_{a}\langle 1|\right) .
\end{aligned}
$$

From (10) and the necessary and sufficient condition of zero discord given by Eq.(3) and (4), we have that state $\rho_{\left|\alpha_{+}\right|}$has zero "right" quantum discord when the components of the "right" reduced density operators are commuting, $\left[\rho_{2}^{A},|0\rangle_{a}\langle 0|\right]=0$. This can be satisfied only when 
$\alpha_{+}=0$ or $\left|\alpha_{+}\right|=1$. Since $\left|\psi_{+}\right\rangle$and $\left|\psi_{-}\right\rangle$are different nonorthogonal states, we do not need to consider those two cases because they corresponds to either the same state or two orthogonal states. Hence as pointed out in Ref. 11], the "right" discord is always non-zero. On the other hand, $\rho_{\left|\alpha_{+}\right|}$has zero "left" quantum discord if and only if $\left[\rho_{1}^{S},|0\rangle\langle 0|\right]=0$, that is,

$$
p_{+}\left(1-\left|\alpha_{+}\right|^{2}\right)=p_{-}\left(1-\left|\alpha_{-}\right|^{2}\right) .
$$

Combining (9) and (11), we have

- $\alpha$ is a real number, and $\alpha \geq 0$;

- $p_{+}=p_{-}=\frac{1}{2}$;

- $\left|\alpha_{+}\right|=\left|\alpha_{-}\right|=\sqrt{|\alpha|}=\sqrt{\alpha}$.

It is interesting that those three conditions coincide exactly with the optimal assisted state discrimination case in 11] with a priori probabilities and $\theta=0$, in which it is shown that the dissonance is required in the discrimination. Thus, we obtain one of our main results: The assisted state discrimination of two nonorthogonal states can be performed with the absence of entanglement. The "right" quantum discord is required for assisted state discrimination. However, the "left" discord is not necessarily to be non-zero. In particular, in the assisted optimal state discrimination, the "left" discord is found to be zero. Here we remark that, it is not surprising that the "left" discord is nonzero except for the optimal case, since quantum discord in general is non-zero [24, 29].

Recall that the motivation of quantum discord is to find the difference between total correlations, including both quantum and classical correlation quantified by mutual information, and the accessible classical correlation, which is quantified by the maximal conditional entropy obtained by local measurement [5]. While the mutual information is symmetric, the asymmetry of the quantum discord is due to local measurements. The protocol of assisted state discrimination in this paper and in Ref. [11], is exactly assisted by local measurements on the "right" party so as to distinguish nonorthogonal states of the "left" party. Thus the corresponding "right" quantum discord is necessary while the "left" discord, which is quantified by a local measurement on the "left" party, is useless. Thus we can find that in the optimal assisted state discrimination, the "left" discord is zero. Indeed, the deep reason that quantum discord is required for assisted state discrimination is that it is really used, as a consuming resource in such quantum information processing.

\section{UNAMBIGUOUS DISCRIMINATION OF $d$ NONORTHOGONAL STATES}

In the following, we generalize the previous model to $d$-dimensional system. Let us consider that a qudit is randomly prepared in the $d(d \geq 2)$ nonorthogonal and linearly independent states, $\left|\psi_{1}\right\rangle,\left|\psi_{2}\right\rangle, \cdots,\left|\psi_{d}\right\rangle$, with a priori probabilities $p_{1}, p_{2}, \cdots, p_{d}$ with $p_{1}+\cdots+p_{d}=1$. The system is coupled to an auxiliary qubit $A$ by a joint unitary transformation $U_{1}$ such that

$$
\begin{aligned}
& U_{1}\left|\psi_{1}\right\rangle|k\rangle_{a}=\sqrt{1-\left|\alpha_{1}\right|^{2}}|1\rangle|0\rangle_{a}+\alpha_{1} \frac{|1\rangle+\cdots+|d\rangle}{\sqrt{d}}|1\rangle_{a}, \\
& U_{1}\left|\psi_{2}\right\rangle|k\rangle_{a}=\sqrt{1-\left|\alpha_{2}\right|^{2}}|2\rangle|0\rangle_{a}+\alpha_{2} \frac{|1\rangle+\cdots+|d\rangle}{\sqrt{d}}|1\rangle_{a}, \\
& U_{1}\left|\psi_{d}\right\rangle|k\rangle_{a}=\sqrt{1-\left|\alpha_{d}\right|^{2}}|d\rangle|0\rangle_{a}+\alpha_{d} \frac{|1\rangle+\cdots+|d\rangle}{\sqrt{d}}|1\rangle
\end{aligned}
$$

where $\{|1\rangle,|2\rangle, \cdots,|d\rangle\}$ is the orthogonal basis in the state space. Note that the auxiliary state is still twodimensional. For $d=2$, we can apply a Hadamard gate on the first qubit, and the model returns to the one given in Section [I] This model is a natural generalization of discrimination of two nonorthogonal states. To ensure that there exists the unitary transformation $U_{1}$, the inner product of vectors on the right hand side should be equal to the overlap of the corresponding original states [12], that is $\alpha_{i}^{*} \alpha_{j}=\left\langle\psi_{i} \mid \psi_{j}\right\rangle$. For convenience, we denote $\alpha_{i j}=\left\langle\psi_{i} \mid \psi_{j}\right\rangle$.

The mixed state we consider in discrimination is given now by

$$
\begin{aligned}
\rho= & p_{1} U_{1}\left(\left|\psi_{1}\right\rangle\left\langle\psi_{1}|\otimes| k\right\rangle_{a}\langle k|\right) U_{1}^{\dagger} \\
& +p_{2} U_{1}\left(\left|\psi_{2}\right\rangle\left\langle\psi_{2}|\otimes| k\right\rangle_{a}\langle k|\right) U_{1}^{\dagger} \\
& +\cdots+p_{d} U_{1}\left(\left|\psi_{d}\right\rangle\left\langle\psi_{d}|\otimes| k\right\rangle_{a}\langle k|\right) U_{1}^{\dagger}
\end{aligned}
$$

The success probability to discriminate the state is given by

$$
P=1-p_{1}\left|\alpha_{1}\right|^{2}-p_{2}\left|\alpha_{2}\right|^{2}-\cdots-p_{d}\left|\alpha_{d}\right|^{2}
$$

In general, it is not zero. If $\rho$ is a separable state, the partial transposed matrix must be positive. Then all the principal minor determinants of $\rho^{T_{S}}$ is non-negative, where $\rho^{T_{S}}$ is the partial transposed matrix with respect to the qudit system. In the following, as a necessary condition for separability, we calculate those $4 \times 4$ principal minor determinants that should be non-negative. Let $M_{i j}$ be the principal minor matrix of $\rho^{T_{S}}$ by selecting the $\langle i| \otimes_{a}\langle 0|,\langle i| \otimes_{a}\langle 1|,\langle j| \otimes_{a}\langle 0|,\langle j| \otimes_{a}\langle 1|$ rows, and the $|i\rangle \otimes|0\rangle_{a},|i\rangle \otimes|1\rangle_{a},|j\rangle \otimes|0\rangle_{a},|j\rangle \otimes|1\rangle_{a}$ columns. By straightforward calculations we have 


$$
M_{i j}=\left(\begin{array}{cccc}
p_{i}\left(1-\left|\alpha_{i}\right|^{2}\right) & p_{i} \alpha_{i}^{*} \sqrt{\frac{1-\left|\alpha_{i}\right|^{2}}{d}} & 0 & p_{j} \alpha_{j}^{*} \sqrt{\frac{1-\left|\alpha_{j}\right|^{2}}{d}} \\
p_{i} \alpha_{i} \sqrt{\frac{1-\left|\alpha_{i}\right|^{2}}{d}} & \frac{1}{d}\left(p_{1}\left|\alpha_{1}\right|^{2}+\cdots+p_{d}\left|\alpha_{d}\right|^{2}\right) & p_{i} \alpha_{i} \sqrt{\frac{1-\left|\alpha_{i}\right|^{2}}{d}} & \frac{1}{d}\left(p_{1}\left|\alpha_{1}\right|^{2}+\cdots+p_{d}\left|\alpha_{d}\right|^{2}\right) \\
0 & p_{i} \alpha_{i}^{*} \sqrt{\frac{1-\left|\alpha_{i}\right|^{2}}{d}} & p_{j}\left(1-\left|\alpha_{j}\right|^{2}\right) & p_{j} \alpha_{j}^{*} \sqrt{\frac{1-\left|\alpha_{j}\right|^{2}}{d}} \\
p_{j} \alpha_{j} \sqrt{\frac{1-\left|\alpha_{j}\right|^{2}}{d}} & \frac{1}{d}\left(p_{1}\left|\alpha_{1}\right|^{2}+\cdots+p_{d}\left|\alpha_{d}\right|^{2}\right) & p_{j} \alpha_{j} \sqrt{\frac{1-\left|\alpha_{j}\right|^{2}}{d}} & \frac{1}{d}\left(p_{1}\left|\alpha_{1}\right|^{2}+\cdots+p_{d}\left|\alpha_{d}\right|^{2}\right)
\end{array}\right) .
$$

The determinant $D_{i j}$ of $M_{i j}$ is given by

$$
\begin{aligned}
D_{i j}= & \operatorname{Det}\left[M_{i j}\right] \\
= & -\frac{1}{d^{2}}\left(p_{i}\left(1-\left|\alpha_{i}\right|^{2}\right)+p_{j}\left(1-\left|\alpha_{j}\right|^{2}\right)\right) \times\left(p_{1}\left|\alpha_{1}\right|^{2}+\cdots\right. \\
& \left.+p_{d}\left|\alpha_{d}\right|^{2}\right) \times\left|p_{i} \alpha_{i} \sqrt{1-\left|\alpha_{i}\right|^{2}}-p_{j} \alpha_{j} \sqrt{1-\left|\alpha_{j}\right|^{2}}\right|^{2} .
\end{aligned}
$$

$D_{i j} \geq 0$ implies that

$$
p_{1} \alpha_{1} \sqrt{1-\left|\alpha_{1}\right|^{2}}=\cdots=p_{d} \alpha_{d} \sqrt{1-\left|\alpha_{d}\right|^{2}} .
$$

In the protocol of assisted state discrimination, $p_{1}, p_{2}, \cdots, p_{d}$ are the priori probabilities. Since the overlap $\alpha_{1 i}=\left\langle\psi_{1} \mid \psi_{i}\right\rangle=\alpha_{1}^{*} \alpha_{i}$ is fixed, without lose of generality, we can rewrite (16) as

$$
\begin{aligned}
p_{1} \alpha_{1} \sqrt{1-\left|\alpha_{1}\right|^{2}} & =p_{2} \frac{\alpha_{12}}{\alpha_{1}^{*}} \sqrt{1-\left|\frac{\alpha_{12}}{\alpha_{1}}\right|^{2}}=\cdots \\
& =p_{d} \frac{\alpha_{1 d}}{\alpha_{1}^{*}} \sqrt{1-\left|\frac{\alpha_{1 d}}{\alpha_{1}}\right|^{2}} .
\end{aligned}
$$

There is only one variable $\alpha_{1}$ in (17) but $d-1$ equalities. By using the condition (16), we successfully write $\rho$ in the following separable form,

$$
\rho=\rho_{1} \otimes|0\rangle_{a}\langle 0|+\rho_{2} \otimes \rho_{a},
$$

where

$$
\begin{aligned}
\rho_{1}= & p_{1}\left(1-\left|\alpha_{1}\right|^{2}\right)|1\rangle\left\langle 1\left|+\cdots+p_{d}\left(1-\left|\alpha_{d}\right|^{2}\right)\right| d\right\rangle\langle d| ; \\
\rho_{2}= & \frac{1}{d}(|1\rangle+\cdots+|d\rangle)(\langle 1|+\cdots+\langle d|) ; \\
\rho_{a}= & \left(p_{1}\left|\alpha_{1}\right|^{2}+\cdots+p_{d}\left|\alpha_{d}\right|^{2}\right)|1\rangle_{a}\langle 1| \\
& +\sqrt{d} p_{1} \sqrt{1-\left|\alpha_{1}\right|^{2}}\left(\alpha_{1}|1\rangle_{a}\left\langle 0\left|+\alpha_{1}^{*}\right| 0\right\rangle_{a}\langle 1|\right) .
\end{aligned}
$$

Thus condition (16) is actually also the sufficient condition for separability.

From the necessary and sufficient condition of zero discord proposed by Eq.(3) and (4), $\rho$ has vanishing "right" discord if and only if $\left[|0\rangle_{a}\langle 0|, \rho_{a}\right]=0$, which gives rise to $\alpha_{1}=0$ or $\alpha_{1}=1$. From (16) it means that the overlap $\left\langle\psi_{i} \mid \psi_{j}\right\rangle$ can only be either 0 or 1 , namely $\left|\psi_{i}\right\rangle,\left|\psi_{j}\right\rangle$ are either orthogonal or equal, which contradicts with our assumption. Therefore we conclude that in assisted state discrimination, the "right" quantum discord is always required. This agrees with the case of two non-orthogonal states.
We consider now the "left" quantum discord. It is easy to find that $\rho_{1}, \rho_{2}$ is linearly independent. Hence $\rho$ has vanishing "left" discord if and only if $\left[\rho_{1}, \rho_{2}\right]=0$. With this commuting condition, i.e., assuming that the "left" discord is zero, we should have

$$
p_{1}\left(1-\left|\alpha_{1}\right|^{2}\right)=\cdots=p_{d}\left(1-\left|\alpha_{d}\right|^{2}\right) .
$$

It means that $\rho_{1}$ is an identity and commutes with any density operators. Combining Eq. (16) and Eq. (19), we obtain

$$
\begin{gathered}
p_{1}=\cdots=p_{d}=\frac{1}{d}, \\
\alpha_{1}=\cdots=\alpha_{d} \equiv \gamma .
\end{gathered}
$$

On the other hand, from the unitary transformation (12), the priori fixed overlaps are equal and should be a real number,

$$
\left\langle\psi_{i} \mid \psi_{j}\right\rangle=\alpha_{i}^{*} \alpha_{j}=|\gamma|^{2} .
$$

To conclude, the "right" discord is always required for the assisted state discrimination of $d(d \geq 2)$ nonorthogonal states, though the quantum entanglement could be absent, i.e. the condition (16) is fulfilled. This is a generalization of assisted state discrimination for two nonorthogonal states [11]. On the other hand, the "left" discord is not necessarily required in this process since conditions (20) and (21) can be fulfilled for some cases which lead also to the absence of entanglement.

As a byproduct, one may notice that the PPT condition is a sufficient condition of separability for state Eq. (13) since it can be written into the form of (18). On the other hand, PPT is a necessary condition for separability. Thus PPT criterion is both the necessary and the sufficient condition for the separability of a class of $2 \times d$ states of the form (13).

\section{OPTIMAL UNAMBIGUOUS DISCRIMINATION OF $d$ NONORTHOGONAL STATES}

Next, we will try to determine the exact form of the optimal success probability given by (14). As $p_{1}, p_{2}, \cdots, p_{d}$ are the priori probabilities and the overlap $\alpha_{1 i}=\left\langle\psi_{1} \mid \psi_{i}\right\rangle=\alpha_{1}^{*} \alpha_{i}$ is known, the success probability 
in (14) can be rewritten as

$$
P=1-p_{1}\left|\alpha_{1}\right|^{2}-\frac{p_{2}\left|\alpha_{12}\right|^{2}+\cdots+p_{d}\left|\alpha_{1 d}\right|^{2}}{\left|\alpha_{1}\right|^{2}} .
$$

There is only one variable $\left|\alpha_{1}\right|$ in (23). In order to find the optimal probability, we define

$$
\bar{\alpha}=\sqrt[4]{\frac{p_{2}\left|\alpha_{12}\right|^{2}+\cdots+p_{d}\left|\alpha_{1 d}\right|^{2}}{p_{1}}} .
$$

The optimal probability can be found by dealing with $\bar{\alpha}$ in three different regions:

1. If $\max \left\{\left|\alpha_{12}\right|, \cdots,\left|\alpha_{1 d}\right|\right\} \leq \bar{\alpha} \leq 1$, then when $\left|\alpha_{1}\right|=$ $\bar{\alpha}$, we have the optimal probability

$$
P=1-2 \sqrt{p_{1}} \sqrt{p_{2}\left|\alpha_{12}\right|^{2}+\cdots+p_{d}\left|\alpha_{1 d}\right|^{2}} .
$$

2. If $\bar{\alpha} \leq \max \left\{\left|\alpha_{12}\right|, \cdots,\left|\alpha_{1 d}\right|\right\}$, then when $\left|\alpha_{1}\right|=$ $\max \left\{\left|\alpha_{12}\right|, \cdots,\left|\alpha_{1 d}\right|\right\}, P$ reaches its optimal point,

$$
\begin{aligned}
P= & 1-p_{1} \max \left\{\left|\alpha_{12}\right|^{2}, \cdots,\left|\alpha_{1 d}\right|^{2}\right\}- \\
& \frac{p_{2}\left|\alpha_{12}\right|^{2}+\cdots+p_{d}\left|\alpha_{1 d}\right|^{2}}{\max \left\{\left|\alpha_{12}\right|^{2}, \cdots,\left|\alpha_{1 d}\right|^{2}\right\}} .
\end{aligned}
$$

3. If $1 \leq \bar{\alpha}$, when $\left|\alpha_{1}\right|=1$, we can obtain the optimal probability

$$
P=1-p_{1}-p_{2}\left|\alpha_{12}\right|^{2}-\cdots-p_{d}\left|\alpha_{1 d}\right|^{2} .
$$

As we have found that the "right" discord is always required for assisted state discrimination, but "left" discord can be zero. In the following, we will show that the "left" discord vanishes in the optimal process.

As we already show that (20) and (21) are the conditions for vanishing "left" discord, in assisted state discrimination, a qudit is randomly prepared in one of the $d$ nonorthogonal states $\left|\psi_{i}\right\rangle(i=1, \cdots, d)$ with an equal priori probability $p_{j}$ and an equal priori and nonzero overlap $\left\langle\psi_{i} \mid \psi_{j}\right\rangle=|\gamma|^{2}$ for all $i \neq j$. Since $\left|\psi_{i}\right\rangle$ is selected in $d$ dimension Hilbert space, this can always be achieved. In this case, the parameter $\bar{\alpha}=\sqrt[4]{d-1}|\gamma|$, and we also have, $\max \left\{\left|\alpha_{12}\right|, \cdots,\left|\alpha_{1 d}\right|\right\}=|\gamma|^{2} \leq \bar{\alpha}$, thus the optimal probability can be expressed as

$$
P_{o p t}= \begin{cases}1-\frac{2 \sqrt{d-1}}{d}|\gamma|^{2}, & 0 \leq|\gamma| \leq \frac{1}{\sqrt[4]{d-1}} \\ \frac{d-1}{d}\left(1-|\gamma|^{4}\right), & \frac{1}{\sqrt[4]{d-1}} \leq|\gamma| \leq 1\end{cases}
$$

We see that the assisted optimal state discrimination can be accomplished with a vanishing "left" discord. Also from (25), we have that for any $d$, the optimal probability is monotonically decreasing with the non-negative parameter $|\gamma|$, i.e., the smaller the overlap is, the larger probability we can discriminate. This is understandable, when they are orthogonal, i.e. the overlap is zero, we can discriminate them deterministically; when they are close to each other, it is difficult to discriminate them. In short, we have shown that the optimal assisted state discrimination of $d(d \geq 2)$ nonorthogonal states can be performed with the absence of entanglement and "left" discord, while the "right" discord is always required.

\section{SUMMARY AND DISCUSSIONS}

It is recently known that besides quantum entanglement, other quantum correlations such as the quantum discord are also useful in quantum information processing. The physical or operational interpretations of quantum discord are still under exploration from different points of view, see e.g. [27, 28]. On the other hand, in the assisted state discrimination [11, it is known that the quantum discord is required. Further in this paper, we find that only the "right" quantum discord is necessary, while the "left" quantum discord can be zero. This clarifies the role of quantum discord in assisted state discrimination. In particular, we find that as a resource for quantum information processing, the use of quantum discord depends on the specified processing task. Explicitly in the process of assisted state discrimination, the measurement on the right hand side is performed, so the "right" quantum discord is necessary. In the absence of entanglement, if one side quantum discord is zero, depending on this specified one side measurement, the quantum correlation of discord is like a classical state. If applied in the assisted state discrimination, it means that the process is like a classical type. Thus the "right" quantum discord is really a useful resource in this process.

Acknowledgments This work is supported by NSFC, "973" program (2010CB922904) and PHR201007107.
[1] M. A. Nielsen and I. L. Chuang, Quantum Computation and Quantum Information (Cambridge University Press, Cambridge, England, 2000).

[2] C. H. Bennett, D. P. DiVincenzo, C. A. Fuchs, T. Mor, E. Rains, P. W. Shor, J. A. Smolin, and W. K. Wootters, Phys. Rev. A 59, 1070 (1999).

[3] E. Knill and R. Laflamme, Phys. Rev. Lett. 81, 5672
(1998).

[4] H. Ollivier, and W. H. Zurek, Phys. Rev. Lett. 88, 017901 (2001).

[5] L. Henderson, and V. Vedral, J. Phys. A 34, 6899 (2001).

[6] J. Oppenheim, M. Horodecki, P. Horodecki, and R. Horodecki, Phys. Rev. Lett. 89, 180402 (2002).

[7] S. Luo, Phys. Rev. A 77, 022301 (2008). 
[8] A. Datta, A. Shaji, and C. M. Caves, Phys. Rev. Lett. 100, 050502 (2008).

[9] A. Peres, Phys. Lett. A 128, 19 (1988), D. Dieks, Phys. Lett. A 126, 303 (1988), I. D. Ivanovic, Phys. Lett. A 123, 257 (1988).

[10] G. Jaeger, and S. Shimony, Phys. Lett. A 197, 83 (1995).

[11] L. Roa, J. C. Retamal, and M. Alid-Vaccarezza, Phys. Rev. Lett. 107, 080401 (2011).

[12] L.-M. Duan and G.-C. Guo, Phys. Rev. Lett. 80, 4999 (1998).

[13] S. Luo, Phys. Rev. A 77, 042303 (2008).

[14] M. D. Lang, and C. M. Caves, Phys. Rev. Lett. 105, 150501 (2010).

[15] M. Ali, A. R. P. Rau, and G. Alber, Phys. Rev. A 81, 042105 (2010).

[16] B. Li, Z. X. Wang and S. M. Fei, Phys. Rev. A 83, 022321 (2011).

[17] Q. Chen, C. Zhang, S. Yu, X. X. Yi, and C. H. Oh, Phys. Rev. A 84, 042313 (2011).

[18] A. Peres, Phys. Rev. Lett. 77, 1413 (1996).

[19] K. Życzkowski, P. Horodecki, A. Sanpera, and M. Lewenstein, Phys. Rev. A 58, 883 (1998).

[20] B. Dakíc, V. Vedral, and Č. Brukner, Phys. Rev. Lett. 105, 190502 (2010).

[21] D. Cavalcanti, L. Aolita, S. Boixo, K. Modi, M. Piani, and A. Winter, Phys. Rev. A 83, 032324 (2011); V. Madhok and A. Datta, Phys. Rev. A 83, 032323 (2011).

[22] D. Girolami and G. Adesso, Phys. Rev. A 83, 052108 (2011).
[23] M. Koashi and A. Winter, Phys. Rev. A 69, 022309 (2004).

[24] A. Ferraro, L. Aolita, D. Cavalcanti, F. M. Cucchietti, and A. Acín, Phys. Rev. A 81, 052318 (2010).

[25] Y. C. Wu and G. C. Guo, Phys. Rev. A 83, 062301 (2011).

[26] M. Shi, W. Yang, F. Jiang, and J. Du, J. Phys. A 44, 415304 (2011).

[27] A. Streltsov, H. Kampermann, and D. Bruss, Phys. Rev. Lett. 106, 160401 (2011).

[28] M. Piani, S. Gharibian, G. Adesso, J. Calsamiglia, P. Horodecki, and A. Winter, Phys. Rev. Lett. 106, 220403 (2011)

[29] A. Streltsov, H. Kampermann, and D. Bruss, Phys. Rev. Lett. 107, 170502 (2011).

[30] G. L. Giorgi, B. Bellomo, F. Galve, and R. Zambrini, Phys. Rev. Lett. 107, 190501 (2011).

[31] M. Gessner, and H-P. Breuer, Phys. Rev. Lett. 107, 180402 (2011).

[32] D. Z. Rossatto, T. Werlang, E. I. Duzzioni, and C. J. Villas-Boas, Phys. Rev. Lett. 107, 153601 (2011).

[33] S. Luo and N. Li, Phys. Rev. A 84, 052309 (2011).

[34] T. Zhou, J. Cui, and G. L. Long, Phys. Rev. A 84, 062105 (2011).

[35] J. Cui, and H. Fan, J. Phys. A 43, 045305 (2010).

[36] K. Modi, T. Paterek, W. Son, V. Vedral, and M. Williamson, Phys. Rev. Lett. 104, 080501 (2010). 